\title{
Digitizing health data for public health protection in the context of European and international coordination
}

\author{
Jennifer Tuzii \\ Department of Legal Science, University of Bologna, Bologna, Italy \\ E-mail: jennifer.tuzii2@unibo.it
}

Received 1 October 2021

Accepted 29 December 2021

\begin{abstract}
.
BACKGROUND: The health sector has long been affected by programs, actions, plans to digitize data and care processes with a view to better protecting individual health, as well as public health, resulting in a slow and uneven development of different and often incompatible national services.

OBJECTIVE: This paper aims to explore the grounds behind the urgency of turning the digital priority into concrete actions, as acknowledged by political leaders in the Rome Declaration, by explaining the capacity of digital tools to enhance healthcare management and the current obstacles.

METHODS: It considers the progressive extension of the EU institutions' scope of action during the pandemic, the related supporting financial strategies launched and some examples of digital contact tracing systems.

RESULTS: It emerged that the pandemic highlighted the inadequacy of purely national policies and the advantages of leveraging the digital health data processing for governance, surveillance and response to cross-border and global threats.

CONCLUSIONS: Considering what emerged during the pandemic and the solemn commitment of the world's major political leaders, the solution to the still existing technical and organizational interoperability issues will no longer be postponed.
\end{abstract}

Keywords: Digitalization, data, public health, surveillance, tracing, interoperability

\section{Health data digitization in European and international health recovery and reform policies}

More than a year after the outbreak of the COVID-19 pandemic and during a phase of progressive recovery of global health and economic systems, taking a perspective of analysis that concerns the protection of public and individual health, it can certainly be considered that the health crisis has shaken global health systems, questioning the way they are organized and function.

Specifically, it has speeded up the process of digitization of health administrations, demonstrating its potential and extreme necessity with regard to the performance of care activities for the treatment of the same condition induced by the contagion; monitoring of contagions; tracing of contacts; and finally data 
sharing between structures and health professionals around the world for the purpose of research and study of the new coronavirus, preparation of prevention protocols in hospitals and care of infected subjects, as well as the development of COVID-19 vaccines.

eHealth refers to a complex and multi-shaped range of health services planned and delivered on the basis of the use of ICT (Information and Communication Technologies), involving the processing of all the health data generated by providers and suppliers with respect to the entire target population through digital devices, to which intelligent processing technologies should theoretically have access. The heterogeneity of health digitization is explained by the existence of functionally diverse systems, such as electronic health records, telemedicine, digital therapies, dematerialized prescriptions, wearable devices, health apps and, outside this diverse category, but supporting it, artificial intelligence. eHealth is a complex phenomenon involving a wide range of different, complementary and coexisting scientific perspectives, from engineering to informatics, from medicine to philosophy, from politics to law. From a legal point of view, the phenomenon takes on further complexity due to the so-called multilateral constitutionalism [1], i.e. a multilevel governance and legal structure made up of local, national, European and international players, as well as the dense network of rules, plans, actions, strategies and the mutual interweaving that results. In this context, another element plays a significant role, namely the necessary incompatibility of the rapid pace of technology with the pace of decision-making and regulation [2].

In this context, an albeit improvised, immature and hasty digitization of health data has allowed, in these critical months, to carry on activities that traditionally relied on tangible economic, commercial, legal, professional and health relations, which were obviously precluded by the bursting diffuseness of a transmissible disease.

On the other hand, a systematic and integrated digital processing of information that public administrations have access to and collect on the basis of an automated system would certainly have made it possible to monitor reliably and more rapidly the spread of the infection, the symptomatic and objective course of the disease in infected individuals and the traceability of the deaths recorded to COVID-19, thus orienting precisely the policies for containing the infection on the basis of information produced in real time according to coherent and synchronic methods of collection and processing from the territories to the state administration.

Most governments around the world have culpably postponed the digital transition of health administrations, now associating the need for it with the outbreak of an emergency that required urgent and now unavoidable investment. Yet digitization is generally the most efficient way to manage the ordinary administration of public health: the reasons lie, on the one hand, in problems caused by the ageing of the population and the increase in chronic diseases, and, on the other, in the need for clinical and organizational appropriateness, related to the shortage of economic resources, and for personalization of care, which is emerging not only because of a more evolved sensitivity and centrality of patients' needs, but also in relation to new gene-based therapies that rely on personalization of care, for example in the field of cancer medicine.

In addition, the application of digital systems to health services would promote effective global access to prevention and treatment, reducing inequalities for people who are unable to physically reach places of treatment for geographical or financial reasons or because they are not self-sufficient. These are difficulties that affect not only developing countries, but also advanced industrialized countries, and which need to be resolved as part of a broader debate that includes the likely side effects of sloppy digitalization [3].

In the opinion of the writer, we need to invest both in the basic digital skills of citizens, who must be able to effectively access the digital channels of health care, since old age cannot preclude the use of the technologies used, as this would cause paradoxical discrimination against the neediest patients; and in 
professional digital skills, since entrusting the use of technologies to health professionals without having previously activated ad hoc training courses is the same as wasting the potential of digital technology.

The COVID-19 emergency has therefore undoubtedly given some real weight to the problems that have long burdened public health administration in almost all developed countries, highlighting their inadequacies, and has therefore given a sense of urgency to actions undertaken over the years in purely programmatic terms, since they have not so far been supported by the real political will to reform health systems.

In this sense and on the basis of what is contained in points 6 and 10, the Rome Declaration, signed in May 2021 by the G20 leaders following the Global Health Summit, constitutes an important signal, formalizing a widely shared political commitment towards the effective digital transformation of health systems and the rapid and secure sharing of scientific data in digital format, functional to the sharing of knowledge, experience and good practices useful for scientific research and epidemiological surveillance on a global scale, as well as for the implementation of preparedness and response systems to international health emergencies.

If what is hoped for is the full integration of ICT in the organization of public health models as well as in the provision of health services to patients, achieving a homogeneous systemization of the technologies that have so far been experimented in a fragmentary manner (the inefficiency of uneven actions is in re ipsa, if we consider that the greatest value of digitalization is the sharing of data at a national, regional and global level), it will not be long before the same need emerges in the private healthcare sector too, because truly achieving the aspiration of patient-centered care requires contiguity and communication between both sectors, given that, albeit to an extremely variable extent, in almost all countries of the world public and private provision coexist.

In the European regional context, which is immediately analyzed through the actions of the European Union, the institutions have made considerable efforts over the last twenty years to build a digital single market, despite their limited competences in health matters and the persistent resistance of the Member States to cede control of their health policy.

The law of the Treaties provides limited scope for the European institutions to act in the field of health, given that the way in which they provide services to their citizens, the organization of health services and access to care form the framework of an exclusively national prerogative, possibly with the support of the Union, which is precluded from any legal harmonization.

The Union has therefore tried the path of voluntary coordination between the Member States, which has so far been insufficient to accompany the digitization of European health systems in an orderly way towards full completion.

By reversing the trend, COVID-19 has led national governments to agree on the irrelevance of geographical and political borders with respect to increasingly global and ontologically widespread phenomena, and on the advisability of coordinated action, to be pursued through strategies and investments that are decisive for the digitization of health data in the Member States and concerted in European and global institutional bodies, through the coordination of the WHO.

This is evident from the shape of the Work Plan [4] drawn up for the implementation of the EU4Health program, launched by the European Commission for the period 2021-2027 and placed within the Next Generation EU, a new instrument that will make it possible to invest total resources amounting to 750 billion euros for the economic recovery of the States affected by the health crisis.

With regard to the public health sector, not only do the Union's economic programs aim to allocate significant resources to strategic assets such as digitization, in order to ensure the resilience of health systems and improve the health of European citizens, but also the governance policies inaugurated, and 
the reform proposals put forward are aimed at strengthening health by leveraging stronger coordination within the Union and with respect to the international context.

Reference is made to the European Health Union [5] project and the European Health Data Space.

As regards the first aspect, the spread of the COVID-19 pandemic has highlighted the close correlation between the health security systems' weakness and the crisis of national economies, guiding the political will of European institutions and Member States towards an urgent process of revision and strengthening of the health governance system, thus motivating the construction of a European Health Union with the aim of strengthening preparedness and response in the event of new health crises.

In fact, the Commission has proposed the adoption of a regulation containing new rules for dealing with health threats of a cross-border nature [6], in order to strengthen emergency preparedness, implement an integrated surveillance system based on digital data sharing, and better manage communication and notification mechanisms between States.

In particular, the Union would be able to declare a public health emergency at EU level and, consequently, adopt measures common to the Member States to deal with future transnational threats, overcoming the limited powers imposed on the institutions in the Treaties and reserving for emergencies an appropriate extraordinary regime, based on closer coordination. The proposal also foresees the establishment of an integrated EU monitoring system for infectious diseases and other health threats.

Further proposals for regulatory reform to broaden the mandate of the ECDC (the European Centre for Disease Prevention and Control) [7], which has so far been relegated to a subsidiary function of collecting data and issuing technical guidance, are also part of the same project; for the strengthening of the pharmacological monitoring, evaluation and surveillance role of the EMA (the European Medicines Agency) in times of crisis [8]; and for the establishment of HERA, a new authority for the preparation and response to health threats, the coordination of biomedical research and development, and the centralized procurement, production and distribution of medicines, vaccines and medical supplies [9].

A further significant expression of the new centrality attributed to the coordinated management of health and health safety through the creation of the European Health Union is the European Health Data Area project, which is cross-cutting in the reform perspectives outlined above.

In this regard, the European Commission has launched a public consultation, open until 26 July 2021, to allow an active participation of European citizens in the project and to solicit concrete proposals to enhance its implementation.

In its communication on a European data strategy, the Commission stresses the need to exploit the potential generated by the developments of the digital technologies available in the health field, in order to improve the processes of prevention, diagnosis and treatment and, in parallel, the mobility of health and the circulation of goods and services within the territory of the Union, through a regulatory framework that in the operations of collection, access, storage, use and re-use of health data in digital format effectively protects the interests and rights of individuals, especially with regard to the processing of sensitive personal health data.

In this context, the Commission also adopted a proposal for a regulation on data governance [10], establishing a robust framework of data access provisions and conditions to build public trust in the voluntary sharing of data for purposes of prophylaxis, diagnosis and treatment, research, and targeted policy and regulatory development.

Ultimately, Europe, as well as the world, is faced with the need to adapt existing tools to radically change the pace and innovate health care and security systems, using digital technology to respond in a rapid, coherent and coordinated way to threats that know no borders, but equally affect governments, societies and economies worldwide. 
After all, if one agrees with the principle that 'when progress is made quickly, sometimes the present resembles a continuous future', one can agree that the problem of communicable diseases and the response represented by digital data processing systems and their continuous technological progress appear to be equally united by the element of speed, coexisting in a single dimension that requires policy makers to act rapidly and coherently.

\section{The digital contribution to epidemiological surveillance activities}

During the response phase to the COVID-19 pandemic, the European Union intended to base its infection containment strategy on digital tracking systems, implicitly affirming the added value of digitizing health data in public health management when threats are so widespread that they cannot be controlled within a defined and controlled physical area. Thus, the use of mobile applications for contact tracing was quickly organized and encouraged to break the chain of infection at national and cross-border level.

In the European institutional context, it is up to the Member States to choose the technical model on the basis of which a tracking app operates, since these are preventive and health management measures, but the adoption of tools that do not comply with European data protection rules is precluded [11].

It is clear that dematerialized processing exposes personal data, sometimes sensitive if they are of a health nature, to greater risks of undue and harmful dissemination than simple manual tracking on paper involving only the citizen and a single person in charge of a public service. Therefore, digital tracking brings with it a complex balancing act between collective health and the individual's right to privacy, which must ensure voluntariness, temporariness and proportionality at the same time, allowing control over the movements of infected persons only to a sufficient extent to achieve the purpose of prevention according to a gradual approach.

The various types of technical operation of applications developed and used to control infection so far essentially relate to two alternative models, centralized and decentralized.

In both cases, the encounter between two parties results in the exchange of encrypted codes between their respective terminal devices using Bluetooth technology; the positive user generates an update on the app.

What differentiates them is the method of storing codes, because in centralized systems data are transmitted to the third-party server managed by the public health administration, while in decentralized apps, which are more inspired by the principle of minimization [12], data are retained by the individual devices of each citizen and only the data of the infected are transferred centrally.

In the first case, the server searches for a match between the codes and sends notifications to those who have come into contact with the infected person, while in the second case it is the individual device that periodically searches the central server for a match between the infected and those intercepted via Bluetooth.

It is clear that decentralized solutions guarantee a higher level of protection of personal data confidentiality that the tracking apps process, so the choice between the two options does not respond so much to technical needs, but is the expression of a specific legal approach.

Italy with Immuni and Germany with Corona-Warn-App are two very similar examples of adherence to a decentralized tracking mechanism, precisely programmed to process the least amount of personal data. However, in the Italian case, little more than $20 \%$ of the population adhered to the contact tracing campaign through the app, and slightly better numbers were recorded in Germany, so the effectiveness of 
this mode of support for traditional health strategies to combat the spread of viruses is compromised by the lack of constant active participation of citizens in the digital tracing process.

Almost everywhere, the coronavirus has led to at least partial restrictions on certain civil freedoms and to substantial changes in the balance of powers and competences, but always within the framework of a balance inspired by the principle of proportionality.

In Hungary, on the other hand, where the political and social situation is already undoubtedly delicate, the government has declared a state of emergency claiming additional powers and margins of intervention, and it is not surprising that, in order to facilitate digital tracking, it has opted for an app based on the centralized model (VirusRadar), unlike almost all the solutions chosen by other European countries.

The different strategy of digital contact tracing expresses a lesser consideration of the individual right to the protection of personal data, which is consistent with the decision to suspend the obligation to comply with the provisions on the protection of personal data outlined in the GDPR (including those referring to the right of access and deletion of personal data) and is set in the context of a peculiar legal sensitivity.

This is demonstrated by the limited degree of permeability of the Hungarian communication policy, given the lack of official information available and the lack of studies on the development and effectiveness of the surveillance and tracing policy followed, which, however, are presumed to be negative given the abandonment of the chosen app. Doubts therefore remain as to the ability of a centralised tracking model to affect infection trends, and the results of a comparison between the two existing methods due to insufficient statistical data.

Intuitively, and subject to the premise of sufficient participation, the centralized option with fewer guarantees should make a significant contribution to the fight against the pandemic, greater than that produced by the decentralized option, and some researchers [13] believe that the mistake was not to bet on the centralized system, flanked by public communication designed not to instil fear in citizens and to clarify the advantages in terms of health.

On the other hand, Far-Oriental legal systems share a legal tradition inspired by opposite values with respect to European liberalism, so applications for population monitoring represent a customary experience, which preserves the primary interest of collective health and ensures a pervasive control regardless of the model chosen.

It is no coincidence that the Beijing government has used apps that are already widely used, and has included a specific tool dedicated to tracking, which is also able to determine the degree of risk of contagion, geolocate the user and send information directly to the police.

The strong governmental meddling in the individual legal dimension of its citizens, the normalization of a condition of continuous and daily surveillance, the collection and aggregation of data and the capillary monitoring of Big Data thus obtained enrich and complete China's digital contact tracing strategy, which has in fact produced excellent results.

For instance, Singapore, which is closely interconnected with mainland China and in particular with the epicenter of the SARS-CoV-2 epidemic, immediately adopted an aggressive response based on the experience of Aviaria and H1N1 and employed the most advanced technologies to keep control and alert systems active, ensuring impressive virtual tracking through a mixed system, but tending to be decentralized, moreover supported by particularly strong restrictive measures and quarantine systems, gaining exemplary results.

These two positive Asian experiences lead us to believe that, regardless of the app model used and apart from the intrusive behavior of the state apparatus in the sphere of the individual freedoms of citizens, which is inconceivable for Western liberal-democratic systems in general but not inseparable from the 
concept of digitization, a complete integration of technologies with the management models of public health is the necessary starting point for an effective policy of contagion containment, on which to build a functioning and guaranteeing system of management and use of the data collected.

Digital recording of administrative data can greatly improve the timely monitoring of the COVID-19 pandemic.

Most countries have classified the number of confirmed cases of infection and the number of deaths as key data, but they classify them through paper-based methods, leading to delays in transmission and reporting and interruptions at weekends and during holidays.

An architecture of digital, intelligent and integrated data collection and processing systems ordinarily used by health and civil protection administrations would have avoided many of these reporting problems.

Another interesting aspect is indirect tracking, involving data collected through other sources outside the public administration, which can play a crucial role in studying the pandemic and predicting other possible outbreaks. Mobility data collected through the use of smartphone apps and reported by platforms such as Facebook, Google, Apple, among others, have been an important input to monitor the drivers of the pandemic in almost all countries. These data sources provide near real-time information on mobility and contacts in almost every country in the world.

However, the fact that we are dealing with sensitive data and that the collectors of these data are private companies opens up the delicate issue of public-private partnerships in data management, as well as the need to make data processing compliant with privacy regulations during implementation, but there is no doubt that the ability to provide huge amounts of near real-time mobility information could be strategic for coordinating future responses.

Although live tracking managed by European governments through specially developed apps that are necessarily privacy-compliant for reasons of transparency of administrative activity has inexplicably been more feared, it remains the most feasible strategy.

However, the usefulness of tracking apps is directly proportional to the diffusion of their use, which implies that the level of protection that apps can provide improves as the number of downloads increases. It has long been argued that contact tracing would only prove effective when an app download threshold of approximately $60 \%$ of the target population is reached.

The identification of this percentage is the result of a relevant English study [14] that, in fact, associates the reliability of the threshold indicated not to the download of the app, but to its use; moreover, for the purposes of producing effects limiting the expansion of the virus, the percentage in question should not be interpreted as a discriminating data, below which the tracing can still determine a benefit for the population. It is clear that mass adherence remains a desirable prospect and should be pursued through a "complex interaction between app adoption in the community, awareness of proximity technologies and public trust' [15].

Large-scale manual contact tracing is however essential and still prevalent in most contexts.

The literature on this topic is particularly diverse and the divergent conclusions reached certainly show the lack of scientific certainty on the real effectiveness of digital contact tracing, but they mainly attribute it to the extreme scarcity of data, collected in short and irrelevant periods of time, and to the difficulty of finding information on the widespread use of tracking apps.

The usefulness of contact tracing apps in combating the COVID-19 pandemic is still under discussion. Some studies seem to provide initial evidence of a positive contribution, both in terms of identifying infected individuals and reducing infection rates, but there are many examples of inadequate use [16]. 


\section{Interoperability as a prerequisite for digitization and coordination in healthcare}

In terms of improving healthcare for patients through telemedicine systems, electronic medical records or digital therapies, as well as in terms of health safety and community protection in the case of communicable diseases, digital systems are the most appropriate and efficient response. However, in order for digitization to become a fully integrated reality in the health data management processes, and to fully express its potential, it is necessary for the active digital systems to operate together, in a coordinated manner, and for the gathered data to be exchanged at a territorial, national, European and international level, without borders and depending on the health need to be satisfied according to an adequacy rationale.

The issue, then, is both legal and technical, since it is a question of data interoperability.

The general definition adopted by the EIF (European Interoperability Framework) describes interoperability of public services as the ability of different organizational systems to interact for the achievement of mutual benefits and common objectives through the sharing of information and knowledge and the exchange of data supported by the respective ICT systems [17]. In short, it is the ability of multiple systems to be interconnected, to exchange information and to be able to use it.

The problem today is not so much the exchange of information, but the ability of individual systems to disseminate information that can be easily understood and used by other points in the network.

Most information systems today are structured according to a "silos" architecture of different applications, which means that they are weakly interconnected, technologically and content-wise heterogeneous, and often not accessible from outside.

Patient data are therefore fragmented and remain within different contexts, with the paradoxical consequence that the objectives of continuity of care, health integration and mobility are thwarted by existing but incompatible technologies, digitized data that cannot be used or interpreted outside the systems that generate them.

More precisely, digital health depends on several types of interoperability. First of all, it requires the simple ability of different technologies - such as a USB stick and a computer - to exchange data, which relates to technical interoperability, which is not particularly problematic today.

To process the exchanged data and extract meaningful and useful information from them, syntactic and semantic interoperability is also required: the former concerns the format and structure of the data that technically arrive at their destination and is ensured by the definition of international standards such as Health Level Seven International (HL7) or Integrating the Healthcare Enterprise (IHE). An emerging standard for healthcare data communication is, for example, HL7's Fast Healthcare Interoperability Resources (FHIR), which defines about 140 common healthcare concepts that can be accessed and exchanged using modern web technologies.

Semantic interoperability, on the other hand, ensures that the meaning of identified medical concepts can be shared between systems, providing a kind of digital common language that is ideally understood by all technologies in the world and by the professionals who manage them to make full use of the information assets in all necessary cases, whether routine or emergency.

At a higher level, there is organizational interoperability, involving political governance and national legislative choices, both of which channel interoperability towards the goal of better healthcare and continuous coordination for the prevention and shared management of cross-border and global threats.

With a view to prevention, it is now clear that artificial intelligence and large-scale analysis of big data will enable the development of health policy strategies based on predictive medicine, precision medicine and the treatment of rare diseases, but the essential prerequisite is the convergence of huge volumes of data 
from different technologies and across geographical and institutional boundaries, overcoming the current difficulties caused by poor or non-existent syntactic and semantic interoperability.

Among other things, the interoperability described above tends to optimally pursue the goals of disease prevention, scientific research and international coordination, which are clearly perceived as crucial during the current and enduring emergency context.

On the research side, fully interoperable data can underpin large-scale observational studies, at national, European and global levels, accelerating institutional coordination for the solution of public health emergencies such as the one experienced through model abstraction and clinical data set correlation systems, thus quickly identifying research or intervention perspectives and enabling multiple teams to conduct different research in parallel at global level [18].

On the other hand, digitization supported by the levels of interoperability described above also has an enormous impact on legal preparedness, as it allows effective epidemiological surveillance systems to be built from the global movements of viral pathogens detected with the help of integrated tracking systems such as those referred to above, so that early action can be taken on emerging outbreaks.

In a world that is globally connected economically, socially, politically and commercially, communicable diseases are an increasingly recurrent threat that needs to be anticipated and immediately isolated, making up for the obvious shortcomings and inconsistencies that have been experienced to date.

\section{Conflict of interest}

None to report.

\section{References}

[1] Di Federico G, Negri S. Unione europea e salute. Principi, azioni, diritti e sicurezza. Milano: Wolters Kluver, CEDAM; 2020.

[2] Miccù R, Questioni attuali intorno alla digitalizzazione dei servizi sanitari nella prospettiva multilivello. In federalismi.it n.5/2021.

[3] Botrugno C. Information and communication technologies in healthcare: A new geography of right to health. Rivista di filosofia del diritto. 2021;X(1).

[4] C(2021) 4793, Annex to the Commission Implementing Decision on the financing of the Programme for the Union's action in the field of health ('EU4Health Programme') and the adoption of the work programme for 2021.

[5] $\operatorname{COM}(2020) 724$, Communication from the Commission to the European Parliament, the Council, the European economic and social committee and the committee of the regions on Building a European Health Union: Reinforcing the EU's resilience for cross-border health threats.

[6] $\operatorname{COM(2020)~727,~Proposal~for~a~Regulation~of~the~European~Parliament~and~of~the~Council~on~serious~cross-border~threats~}$ to health and repealing Decision No 1082/2013/EU.

[7] $\operatorname{COM}(2020) 726$, Proposal for a Regulation of the European Parliament and of the Council amending Regulation (EC) No $851 / 2004$ establishing a European Centre for disease prevention and control.

[8] $\operatorname{COM(2020)~725,~Proposal~for~a~Regulation~of~the~European~Parliament~and~of~the~Council~on~a~reinforced~role~for~the~}$ European Medicines Agency in crisis preparedness and management for medicinal products and medical devices.

[9] COM(2021) 577, Proposal for a Council Regulation on a framework of measures for ensuring the supply of crisis-relevant medical countermeasures in the event of a public health emergency at Union level.

[10] COM (2020) 767, Proposal for a regulation of the European Parliament and of the Council on European Data Governance (Data Governance Act).

[11] Reg. UE 679/2016, General Data Protection Regulation (GDPR).

[12] Art. 5, Reg. UE 679/2016.

[13] White L, van Basshuysen P. Privacy versus public health? A reassessment of centralised and decentralised digital contact tracing. Science and Engineering Ethics. 2021;27:23. 
[14] Available from: https://www.research.ox.ac.uk/article/2020-04-16-digital-contact-tracing-can-slow-or-even-stop-corona virus-transmission-and-ease-us-out-of-lockdown.

[15] Grekousis G, Liu Y. Digital contact tracing, community uptake, and proximity awareness technology to fight COVID-19: A systematic review In: Sustainable Cities and Society 2021.

[16] Chiusi F, Digital contact tracing apps: Do they actually work? A review of early evidence. In algorithmwatch.org, 8 luglio 2021.

[17] COM (2010) 744.

[18] Lehne M, Sass J, Essenwanger A, Thun S. Why digital medicine depends on interoperability. npj Digital Medicine. 2019;279. 